Boundedness of the mixed velocity-temperature derivative skewness in homogeneous isotropic turbulence

S. L. Tang', R. A. Antonia, L. Djenidi, L. Danaila, and Y. Zhou

Citation: Physics of Fluids 28, 095102 (2016); doi: 10.1063/1.4961466

View online: http://dx.doi.org/10.1063/1.4961466

View Table of Contents: http://aip.scitation.org/toc/phf/28/9

Published by the American Institute of Physics

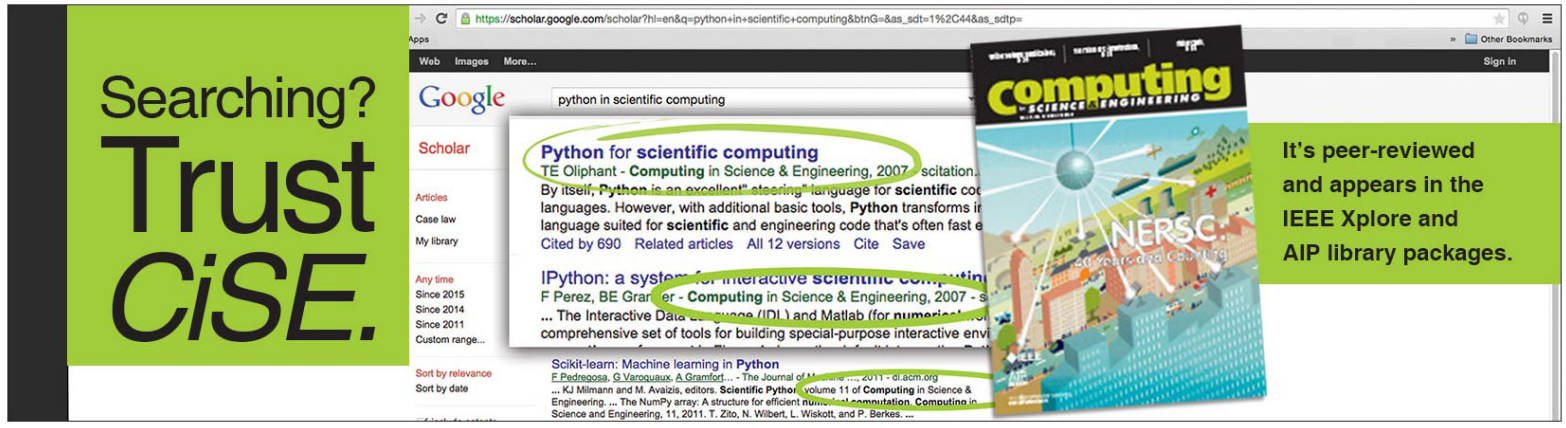




\title{
Boundedness of the mixed velocity-temperature derivative skewness in homogeneous isotropic turbulence
}

\author{
S. L. Tang, ${ }^{1, a)}$ R. A. Antonia, ${ }^{2}$ L. Djenidi, ${ }^{2}$ L. Danaila, ${ }^{3}$ and Y. Zhou ${ }^{1,4}$ \\ ${ }^{1}$ Institute for Turbulence-Noise-Vibration Interaction and Control, Shenzhen Graduate \\ School, Harbin Institute of Technology, Shenzhen 518055, People's Republic of China \\ ${ }^{2}$ School of Engineering, University of Newcastle, Newcastle NSW 2308, Australia \\ ${ }^{3}$ CORIA CNRS UMR 6614, Université de Rouen, 76801 Saint Etienne du Rouvray, France \\ ${ }^{4}$ Digital Engineering Laboratory of Offshore Equipment, Shenzhen 518055, \\ People's Republic of China
}

(Received 20 April 2016; accepted 9 August 2016; published online 1 September 2016)

\begin{abstract}
The transport equation for the mean scalar dissipation rate $\bar{\epsilon}_{\theta}$ is derived by applying the limit at small separations to the generalized form of Yaglom's equation in two types of flows, those dominated mainly by a decay of energy in the streamwise direction and those which are forced, through a continuous injection of energy at large scales. In grid turbulence, the imbalance between the production of $\bar{\epsilon}_{\theta}$ due to stretching of the temperature field and the destruction of $\bar{\epsilon}_{\theta}$ by the thermal diffusivity is governed by the streamwise advection of $\bar{\epsilon}_{\theta}$ by the mean velocity. This imbalance is intrinsically different from that in stationary forced periodic box turbulence (or SFPBT), which is virtually negligible. In essence, the different types of imbalance represent different constraints imposed by the large-scale motion on the relation between the so-called mixed velocity-temperature derivative skewness $S_{T}$ and the scalar enstrophy destruction coefficient $G_{\theta}$ in different flows, thus resulting in non-universal approaches of $S_{T}$ towards a constant value as $R e_{\lambda}$ increases. The data for $S_{T}$ collected in grid turbulence and in SFPBT indicate that the magnitude of $S_{T}$ is bounded, this limit being close to 0.5. Published by AIP Publishing. [http://dx.doi.org/10.1063/1.4961466]
\end{abstract}

\section{INTRODUCTION}

It is generally accepted that the skewness $S$ of the longitudinal velocity derivative, usually defined as

$$
S=\frac{\overline{(\partial u / \partial x)^{3}}}{\overline{(\partial u / \partial x)^{2}}}
$$

( $u$ is the longitudinal velocity fluctuation, $x$ is in the flow direction; the overbar denotes time averaging for experimental data and space/time averaging for numerical data), is an important quantity in turbulence research since it is closely linked to the production of the mean energy dissipation rate caused by vortex stretching. Similarly, the mixed velocity-temperature derivative skewness $S_{T}$, defined as

$$
S_{T}=\frac{\overline{(\partial u / \partial x)(\partial \theta / \partial x)^{2}}}{{\overline{(\partial u / \partial x)^{2}}}^{1 / 2} \overline{(\partial \theta / \partial x)^{2}}}
$$

( $\theta$ is the instantaneous temperature fluctuation), is also important since it represents the production of $\bar{\epsilon}_{\theta}$ due to the stretching of the temperature field by the turbulent strain field. ${ }^{1}$ As a result of

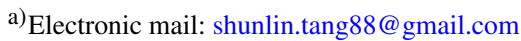


the first similarity hypothesis by Kolmogorov ${ }^{2}$ and the corresponding hypothesis of Obukhov, ${ }^{3}$ one expects that $S$ and $S_{T}$ should both become constant when the Taylor microscale Reynolds number $\operatorname{Re}_{\lambda}\left(=u^{\prime} \lambda / v\right.$, where $\lambda$ is the longitudinal Taylor microscale $u^{\prime} /(\partial u / \partial x)^{\prime}$ and $v$ is the kinematic viscosity of the fluid, a prime denotes a rms value) is sufficiently large. The second similarity hypothesis of Kolmogorov ${ }^{4}$ and Obukhov, ${ }^{5}$ together with the 4/5 law ${ }^{6}$ and the 4/3 law $^{7}$ (see also Monin and $\left.\mathrm{Yaglom}^{8}\right)$ predicts that the skewness of $\delta u(\delta u$ is the velocity increment $u(x+r)-u(x)$ between two points separated by a distance $r$ ), viz., $S_{\delta u}=\overline{(\delta u)^{3}} /{\overline{(\delta u)^{2}}}^{3 / 2}$, and the mixed skewness $S_{\delta u(\delta \theta)^{2}}=\overline{\delta u(\delta \theta)^{2}} /\left({\overline{(\delta u)^{2}}}^{1 / 2} \overline{(\delta \theta)^{2}}\right)$ should become constant within the inertial range $(\delta \theta$ is the temperature increment $\theta(x+r)-\theta(x))$. The combinations of the two hypotheses by Kolmogorov ${ }^{2}$ and Obukhov $^{3}$ are usually referred to in the literature as K41 \& O49. The third similarity hypothesis, introduced by Kolmogorov ${ }^{4}$ (K62) to account for the spatio-temporal fluctuations of the instantaneous energy dissipation rate $\epsilon$, and the corresponding hypothesis for a passive scalar advected by a locally homogeneous isotropic turbulence ${ }^{5}$ (O62) allow $S$ and $S_{T}$ to vary with $R e_{\lambda}$, even when the latter is very large. Concomitantly, $S_{\delta u}$ and $S_{\delta u(\delta \theta)^{2}}$ need not be constant within the inertial range. Predictions in the inertial range remain a major challenge in turbulence, especially in decaying type flows, e.g., grid turbulence, jets and wakes, where extremely high values of $R e_{\lambda}$ are needed to establish the inertial range. ${ }^{9}$ There is now significant evidence ${ }^{9-13}$ that particular attention needs to be paid to the finite Reynolds number effect when assessing the scaling range behavior of the velocity structure functions before drawing any conclusion regarding the validity of the anomalous scaling predicted by K62. Similarly, we expect the FRN effect to influence the statistics of passive scalars in the scaling range since very high values of $R e_{\lambda}$ are required before the inertial range is established, the focus in this paper is on the statistics of $S_{T}$, a quantity associated with the dissipative range. With a few exceptions, most experimental and numerical results reported in the literature indicate a slow but continuous increase of $|S|$ and $\left|S_{T}\right|$ with $R e_{\lambda}$, viz.,

$$
\begin{array}{cc}
|S| \sim \operatorname{Re}_{\lambda}^{\alpha} & (\alpha>0), \\
\left|S_{T}\right| \sim \operatorname{Re}_{\lambda}^{\beta} & (\beta>0),
\end{array}
$$

especially if atmospheric surface layer data are included, e.g., work of Sreenivasan and Antonia, ${ }^{14}$ Warhaft, ${ }^{15}$ and Chassaing et al. ${ }^{16}$

In the case of $|S|$, Antonia et al. ${ }^{17}$ showed that when the atmospheric surface layer data are disregarded (a compelling reason for doing so is that these data were obtained at a relatively small height above the ground or ocean surface, i.e., under conditions that are unlikely to comply with those prescribed by K41), the bulk of the laboratory data (e.g., Fig. 5 of Ref. 14) does not exclude the possibility that $|S|$ becomes constant when $R e_{\lambda}$ approaches $10^{3}$. Since the laboratory data have been collected from a wide range of different flows, $|S|$ may be flow-dependent. Indeed, in a recent paper, Thiesset, Antonia, and Djenidi ${ }^{18}$ showed that along the axis of the self-preserving far-field of a turbulent round jet, the transport equation for the mean kinetic energy dissipation rate $\bar{\epsilon}$, defined by

$$
\bar{\epsilon}=v \overline{\left(\frac{\partial u_{i}}{\partial x_{j}}+\frac{\partial u_{j}}{\partial x_{i}}\right) \frac{\partial u_{j}}{\partial x_{i}}} \quad(i, j=1,2,3)
$$

( $u_{i}$ are the velocity fluctuations in the $x_{i}$ directions), imposes a fundamental constraint on the balance between $S$ and the enstrophy destruction coefficient $G$, defined by

$$
G=\overline{u_{1}^{2}} \frac{\overline{\left(\partial^{2} u_{1} / \partial x_{1}^{2}\right)^{2}}}{\overline{\left(\partial u_{1} / \partial x_{1}\right)^{2}}},
$$

which depends on the type of large-scale forcing and may thus differ from flow to flow. Note that, in this paper, $u_{1}, u_{2}$, and $u_{3}$ will be used interchangeably with $u, v, w$, similarly for $x_{1}, x_{2}, x_{3}$ and $x, y, z$. Tang et al. ${ }^{19,20}$ and Antonia et al. ${ }^{17}$ (hereafter, we denote these three papers collectively as SA15) further showed that the magnitude of $S$ in various turbulent flows, e.g., grid turbulence, along the axis of the self-preserving far-field of a round jet, along the centreline of a fully developed channel flow, on the centreline of the far-wake of a circular cylinder, and stationary forced periodic 
box turbulence (SFPBT), has an upper bound, a value slightly larger than 0.5 , while the path along which $S$ approaches this universal constant is flow dependent. This is consistent with $K 41$ and also the prediction of Qian, ${ }^{21}$ but in contradiction to the modified similarity hypothesis of K62. SA15 further pointed out that the constancy of $|S|$ at sufficiently large $R e_{\lambda}$, which contrasts with (3), should not be too surprising. They argued that the constancy of $S$ is consistent with the constraint imposed by the transport equation for $\bar{\epsilon}$ and the universal collapse of the high wavenumber part of the Kolmogorov-normalized $u$-spectrum. ${ }^{22}$

For $\left|S_{T}\right|$, one expects that (i) the transport equation for $\bar{\epsilon}_{\theta}$, defined by

$$
\bar{\epsilon}_{\theta}=\kappa \overline{\left(\frac{\partial \theta}{\partial x_{i}}\right)^{2}}
$$

should also impose a fundamental constraint on the balance between $S_{T}$ and the destruction coefficient of the scalar dissipation rate, defined by

$$
G_{\theta}=\overline{\theta^{2}} \frac{\overline{\left(\partial^{2} \theta / \partial x^{2}\right)^{2}}}{{\overline{(\partial \theta / \partial x)^{2}}}^{2}},
$$

(ii) this constraint may depend on the nature of the flow; (iii) $\left|S_{T}\right|$ may approach a universal constant when $R e_{\lambda}$ is sufficiently large, although the way this constant is approached will most likely depend on the flow. The present work aims to provide some insight into the transport equation for $\bar{\epsilon}_{\theta}$ in grid turbulence and SFPBT and draw attention to the constraint it imposes on the evolution of $\left|S_{T}\right|$ as $R e_{\lambda}$ increases. Furthermore, although the experimental data in Fig. 9 of Ref. 14 (this will be discussed in some detail at the end of Section III) show a continuous increase of $\left|S_{T}\right|$ with $R e_{\lambda}$ when the atmospheric surface layer data are included, they also indicated that the DNS data of Kerr ${ }^{23}$ exhibit only a marginal dependence on $R e_{\lambda}$ with a value of about 0.5 . Sreenivasan and Antonia ${ }^{14}$ pointed out that "the discrepancy in the magnitude of $S_{T}$ between the experiments and Kerr's (1985) simulations needs to be resolved." This issue is also addressed and resolved in the present paper.

\section{THEORETICAL CONSIDERATIONS}

To carry out the analysis we use the same theoretical framework used by SA15 and which is based on the scale-by-scale energy budget or the transport equation of $\overline{(\delta u)^{2}}$. We start with the generalized form of Yaglom's equation in grid turbulence, ${ }^{24}$

$$
-\frac{U}{r^{2}} \int_{0}^{r} s^{2}\left[\frac{\partial \overline{(\delta \theta)^{2}}}{\partial x}\right] d s-\overline{\delta u(\delta \theta)^{2}}+2 \kappa \frac{\partial}{\partial r} \overline{(\delta \theta)^{2}}=\frac{4}{3} \bar{\epsilon}_{\theta} r,
$$

where $\kappa$ is the thermal diffusivity; and $\bar{\epsilon}_{\theta}=3 \kappa \overline{(\partial \theta / \partial x)^{2}}$ stands for the isotropic mean temperature dissipation rate. Eq. (8) reduces to

$$
\frac{1}{2} U \frac{\partial \overline{\theta^{2}}}{\partial x}+\bar{\epsilon}_{\theta}=0
$$

when $r$ exceeds the integral scale $L$. When $r \rightarrow 0$, the transport equation for $\bar{\epsilon}_{\theta}$ simplifies to

$$
-\frac{U}{15 \kappa} \frac{\partial \bar{\epsilon}_{\theta}}{\partial x}=\overline{\frac{\partial u}{\partial x}\left(\frac{\partial \theta}{\partial x}\right)^{2}}+\frac{2}{3} \kappa \overline{\left(\frac{\partial^{2} \theta}{\partial x^{2}}\right)^{2}} .
$$

This can be rewritten as

$$
-U \frac{\partial \bar{\epsilon}_{\theta}}{\partial x}=\left(\frac{5}{3}\right)^{1 / 2} \frac{\bar{\epsilon}_{\theta} \bar{\epsilon}^{1 / 2}}{v^{1 / 2}}\left[S_{T}+\frac{2 \sqrt{15}}{9} \frac{G_{\theta}}{P e} \operatorname{Pr}^{1 / 2} R_{\theta}^{-1 / 2}\right],
$$

where $P r=v / \kappa$ is the Prandtl number; $P e$ is the turbulent Peclet number,

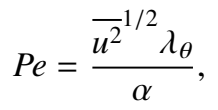


with the Corrsin microscale $\lambda_{\theta}$ defined as $\left({\overline{\theta^{2}}}^{1 / 2} /{\overline{(\partial \theta / \partial x)^{2}}}^{1 / 2}\right)$ and $R_{\theta}$ is the time scale ratio,

$$
R_{\theta}=\frac{\overline{\theta^{2}} / \bar{\epsilon}_{\theta}}{\overline{q^{2}} / \bar{\epsilon}} .
$$

If the grid turbulence has an imposed mean temperature gradient, an additional production term due to the mean temperature gradient should be accounted for in the transport equation for $\bar{\epsilon}_{\theta}$; this has been discussed in some detail in the work of Danaila and Mydlarski. ${ }^{25}$ Also, we should stress that Eq. (8) is derived from the heat transport equation and is valid for any value of $\operatorname{Pr}$. Thus, Eq. (11), which is obtained from Eq. (8) when $r \rightarrow 0$, is also tenable for any $P r$. Here, we focus only on the case $\operatorname{Pr} \approx 1$ in grid turbulence since the available experiments in the literature were conducted at $\operatorname{Pr}=0.7$.

If a power-law decay rate for $\overline{\theta^{2}}$ is assumed in grid turbulence, viz., $\overline{\theta^{2}} \sim x^{-m}$, then (11) can be rewritten as

$$
S_{T}+\frac{2 \sqrt{15}}{9} \frac{G_{\theta}}{P e} \operatorname{Pr}^{1 / 2} R_{\theta}^{-1 / 2}=\frac{C_{\theta}}{R e_{\lambda}}
$$

where

$$
C_{\theta}=2\left(\frac{m+1}{n}\right)
$$

Some comments on recent results concerning the decay of grid turbulence are required before continuing the analysis. Applying a self-preservation analysis to the scale-by-scale energy budget equation, Djenidi and Antonia ${ }^{26}$ showed that a power-law decay is tenable strictly when $R e_{\lambda}$ is constant during the decay. This corresponds to $n=1$ and self-preservation is satisfied at all scales of motion. They also showed that, for any value of $n>1, R e_{\lambda}$ decreases with $x$. Djenidi, Kamruzzaman, and Antonia ${ }^{27}$ further showed that for most grid turbulence measurements, the turbulence decay is in a transition period, which has been invariably mistaken for the initial period, and that the decay is best described by a family of power-laws $\overline{u^{2}} \sim x^{-n_{i}}$, where the exponent $n_{i}$ is only valid for a short distance over which one can assume $R e_{\lambda}$ to be approximately constant. One expects that a similar conclusion can be reached for the decay of the passive scalar in grid turbulence. It is worth recalling here the argument of Speziale and Bernard ${ }^{28}$ that $n=1$ is "the asymptotic state toward which self-preserving isotropic turbulence is driven at high Reynolds numbers in order to resolve the fundamental imbalance between vortex stretching and viscous diffusion. In the process of resolving this imbalance, compatibility with Kolmogorov scaling is achieved for the small-scale correlations." On the other hand, a variation of $n$ and $m$ with $R e_{\lambda}$ does not significantly affect the estimations of $C / R e_{\lambda}$ and $C_{\theta} / R e_{\lambda}$ when $R e_{\lambda}$ is sufficiently large, e.g., at $R e_{\lambda}=300, C_{\theta} / R e_{\lambda}$ ( or $C / R e_{\lambda}$ ) is 0.012 and 0.013 if $n=m=1.2$ and $n=m=1.0$, respectively.

The above discussion clearly indicates that the paths that both $\left(S_{q}+\frac{2 \sqrt{15}}{9} \frac{G_{q}}{R e_{\lambda}} R^{-1 / 2}\right)$ and $\left(S_{T}+\frac{2 \sqrt{15}}{9} \frac{G_{\theta}}{P e} \operatorname{Pr}^{1 / 2} R_{\theta}^{-1 / 2}\right)$ follow to reach zero depend on the values of $n$ and $m$. For convenience, we use $n=m=1.2$ to be consistent with the experiment of Lee et al. ${ }^{29}$

With regard to SFPBT, where the forcing is usually concentrated at very low wavenumbers (i.e., very large scales), one can expect that the magnitude of $C$, although not necessarily zero, is smaller than in either decaying turbulence or in turbulent flows where the forcing is spread over a range of scales. For example, the estimated ratio of the large scale forcing term $\left(C / R e_{\lambda}\right)$ to $S$ is about 0.016 for the SFPBT of Fukayama $e t a l .^{30}$ at $R e_{\lambda}=70$ (estimated from their Fig. $5)$ and is several orders of magnitude smaller for the SFPBT of Gotoh, Fukayama, and Nakano ${ }^{31}$ at $R e_{\lambda}=460$. In this case, $S+2 G / R_{\lambda}$ should be very nearly zero, as pointed out by SA15. It is reasonable to assume that $\left(C_{\theta} / R e_{\lambda}\right)$ is also nearly zero when a passive scalar is involved.

\section{RESULTS}

The above analytical considerations point to the sum $\left(S_{T}+\frac{2 \sqrt{15}}{9} \frac{G_{\theta}}{P e} \operatorname{Pr}^{1 / 2} R_{\theta}^{-1 / 2}\right)$ approaching zero at sufficiently large $R e_{\lambda}$ in grid turbulence. This is indeed suggested by Fig. 1, which shows the 


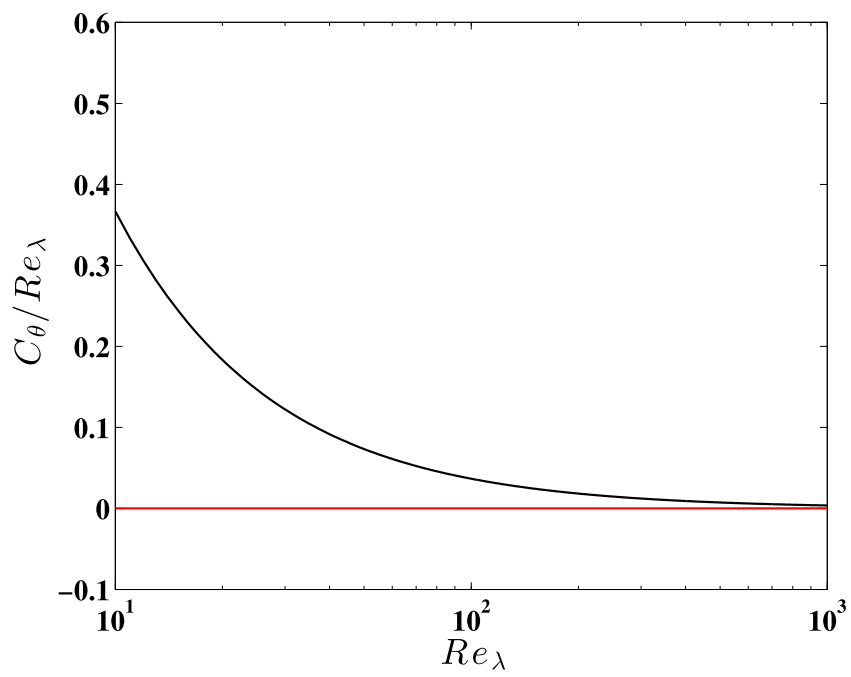

FIG. 1. Dependences of $C_{\theta} / R e_{\lambda}$ on $R e_{\lambda}$ in grid turbulence (black) with $C_{\theta}=2\left(\frac{m+1}{n}\right)$ (Eq. (15)) $(n=m=1.2)$ and in SFPBT (red).

variation of $C_{\theta} / R e_{\lambda}$ in terms of $R e_{\lambda}$ for both grid turbulence and SFPBT. Considering that $C_{\theta} / R e_{\lambda}$ for SFPBT should lie close to zero and given the uncertainties (e.g., noise contamination and/or inadequate spatial resolution in hot-wire measurements) in estimating $S_{T}$ and $G_{\theta}$, it is unlikely that one will ever be able to distinguish unambiguously the departure of $\left(S_{T}+\frac{2 \sqrt{15}}{9} \frac{G_{\theta}}{P e} \operatorname{Pr}^{1 / 2} R_{\theta}^{-1 / 2}\right)$ from zero between grid turbulence and SFPBT when $R e_{\lambda} \geq 300$. On the other hand, as shown below, one may be able to estimate the second term of this expression, even at large Reynolds numbers.

The term $G_{\theta}$, defined by (7), can be rewritten as

$$
G_{\theta}=\frac{\overline{\theta^{* 2}} \overline{\left(\partial^{2} \theta^{*} / \partial x^{* 2}\right)^{2}}}{\overline{\left(\partial \theta^{*} / \partial x^{*}\right)^{2}}},
$$

where the asterisk denotes normalization by the Obukhov-Corrsin scales, ${ }^{3,32}$ or equivalently, for $\operatorname{Pr}=1$, the Batchelor scales: $\theta_{B}=\left(\bar{\epsilon}_{\theta}(v / \bar{\epsilon})^{1 / 2}\right)^{1 / 2}$ and $\eta_{B}=\eta \operatorname{Pr}^{-1 / 2}$. In this paper, we use the Batchelor scales since they lead to a reasonable collapse of the scalar spectra over a large range of $\operatorname{Pr}^{33-35}$ Thus, the second term on the left of (14) can be rewritten as

$$
\frac{2 \sqrt{15}}{9} \frac{G_{\theta}}{P e} \operatorname{Pr}^{1 / 2} R_{\theta}^{-1 / 2}=2 \sqrt{15} \int_{0}^{\infty} k_{1}^{* 4} \phi_{\theta}^{*}\left(k_{1}^{*}\right) d k_{1}^{*},
$$

where $\phi_{\theta}^{*}\left(k_{1}^{*}\right)$ is the one-dimensional spectral density of $\theta$, defined such that $\int_{0}^{\infty} \phi_{\theta}\left(k_{1}\right) d k_{1}=\overline{\theta^{2}}$.

There is ample evidence in the literature to indicate that the integral in (17) converges rapidly to a constant value with increasing $R e_{\lambda}$. For example, Lee et al. ${ }^{29}$ used the grid turbulence data for estimating $G_{\theta} / R e_{\lambda}$ with $R e_{\lambda}$ in the range 10-1360. They showed that the ratio $G_{\theta} / R e_{\lambda}$ approaches a constant relatively rapidly with increasing $R e_{\lambda}$. This constancy of $G_{\theta} / R e_{\lambda}$ reflects the collapse of $E_{\theta}^{*}\left(k^{*}\right)\left(E_{\theta}(k)\right.$ is the 3D scalar spectrum) or $\phi_{\theta}\left(k_{1}\right)$ onto a single curve at all wavenumbers when normalized with $\theta_{B}$ and $\eta_{B}$. We report in Figs. 2 and 3 the distributions $k^{* 4} E_{\theta}^{*}\left(k^{*}\right)$ and $k_{1}^{* 4} \phi_{\theta}^{*}\left(k_{1}^{*}\right)$, respectively, collected in several turbulent flows. In Fig. 2, 3D spectra from direct numerical simulations of non-isothermal HIT are shown, while Fig. 3 contains measurements in grid turbulence, the wake centreline as well as numerical results for SFPBT and channel flow. It can be seen from Fig. 2, where $88 \leq R e_{\lambda} \leq 529$, that, notwithstanding the peeling off of some distributions due to the residual aliasing errors, there is a very good collapse for $E_{\theta}^{*}\left(k^{*}\right)$, illustrating the constancy of $G_{\theta} / R e_{\lambda}$. A similar observation can be made for the experimental data reported in Fig. 3, which also reports some numerical simulation results (in that figure, $30 \leq R e_{\lambda} \leq 195$ ). The distributions of $k_{1}^{* 4} \phi_{\theta}^{*}\left(k_{1}^{*}\right)$ exhibit a reasonable collapse despite the small scatter in the distributions and the peeling off of one distribution. Consequently, the collapse in Figs. 2 and 3, in accord with Batchelor scaling, 


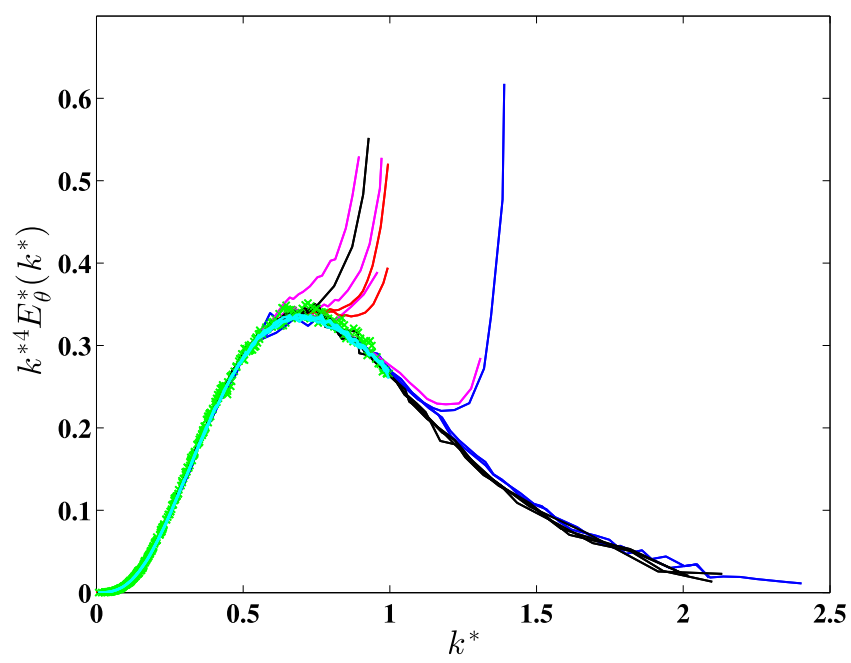

FIG. 2. Normalized three-dimensional scalar spectra $k^{* 4} E_{\theta}^{*}\left(k^{*}\right)$. Blue curves, Donzis, Sreenivasan, and Yeung, ${ }^{35} R e_{\lambda}=240$ with different resolutions ( $k_{\max }=1.4 \sim 5.14$ ). Pink curves, Gotoh and Watanabe, ${ }^{36} R e_{\lambda}=174,263,468$, and 586 respectively. Red curves, Watanabe and Gotoh, ${ }^{37} R e_{\lambda}=258$ and 427 respectively. Black curves, Watanabe and Gotoh, ${ }^{38} \operatorname{Re}_{\lambda}=176,178$, 180,414 and 427 respectively. Green symbols, Wang, Chen, and Brasseur, ${ }^{39} R e_{\lambda}=100,151$, and 195 respectively. Light blue curves, Gauding, ${ }^{40} \operatorname{Re}{ }_{\lambda}=88,119,184215,331$, and 529 respectively.

underpins the constancy of the integral term of Eq. (17). Interestingly, the value of the constant appears to be flow independent, which in turn indicates that the right side of Eq. (17) is also flow independent. To estimate the integral term of Eq. (17), a uniform treatment was applied to the distributions of $k_{1}^{* 4} \phi_{\theta}^{*}\left(k_{1}^{*}\right)$ (Fig. 3), primarily to avoid the possibility of an inadequate spatial resolution in the experiment (hot- and cold-wire measurements) and simulations; this treatment is similar to that used for estimating $2 G / R e_{\lambda}$ in SA15. To carry out the integration, we applied a curve-fit to the data in the region $\phi_{\theta}\left(k_{1}^{*}\right)$ beyond $k_{1}^{*} \approx 0.72$. The curve-fit is based the model spectrum,

$$
\phi_{\theta}^{*}\left(k_{1}^{*}\right)=C_{s p} \exp \left\{-\beta\left\{\left[\left(k_{1}^{*}\right)^{4}+c_{\eta}^{4}\right]^{1 / 4}-c_{\eta}\right\}\right\},
$$

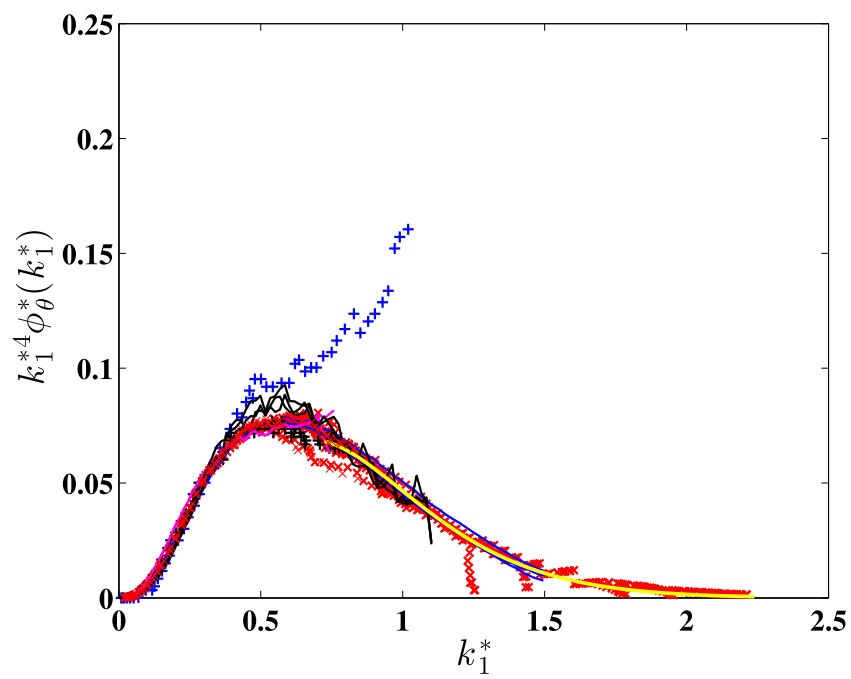

FIG. 3. Normalized one-dimensional scalar spectra $k_{1}^{* 4} \phi_{\theta}^{*}\left(k_{1}^{*}\right)$. (a) grid turbulence: blue plus, Antonia et al.; $;^{41}+$, Lee et al. $;^{42}$ (b) SFPBT: red times, Ref. 39; (c) wake centreline: pink curves, Ref. 43; black curves, Ref. 44 (the true $\bar{\epsilon}$ is used); blue curves correspond to spectra on the channel centreline. ${ }^{45}$ The yellow curve is calculated from Eq. (18). 


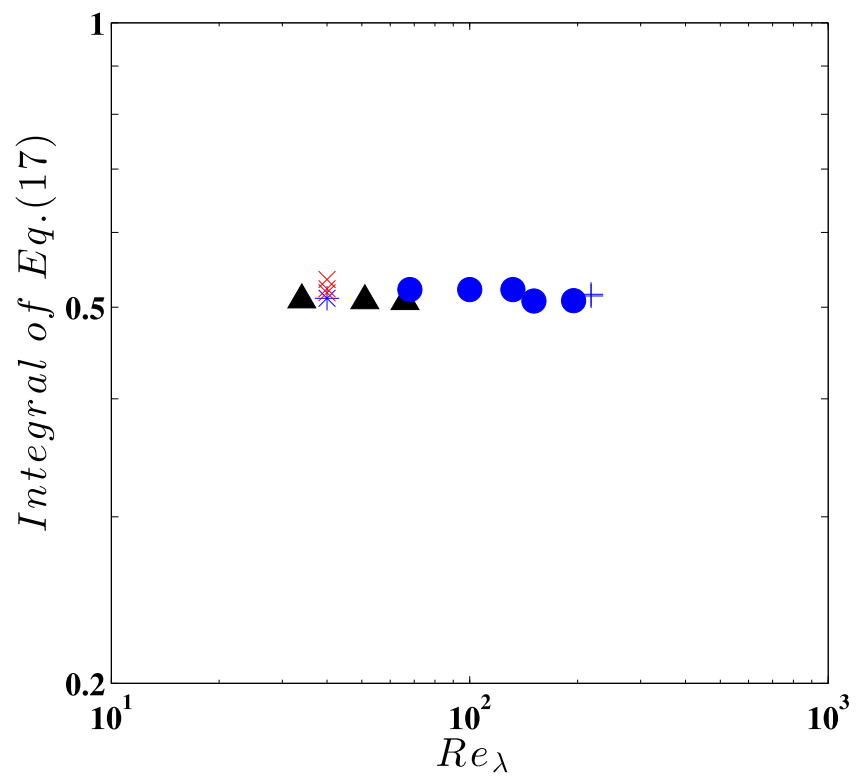

FIG. 4. Integral of Eq. (17). *, Ref. 42; blue filled circle, Ref. 39; blue plus, Ref. 43; red times, Ref. 44; black filled bigtriangleup, Ref. 45.

with $C_{s p}=1 / 400000, c_{\eta}=0.35$ and $\beta=6.2$. We should stress that the very good collapse of the data in the range of interest (i.e., $k_{1}^{*} \geq 0.72$ see Fig. 3) implies that the model spectrum (Eq. (18)) represents a curve fit to all spectra. The reasonable collapse of the scalar spectra over a large range of $\operatorname{Pr}^{33-35}$ using the Batchelor scales justifies the use of a universal extrapolation. The results of the integration of $k_{1}^{* 4} \phi_{\theta}^{*}\left(k_{1}^{*}\right)$ are shown in Fig. 4 for several flows. The numerical value of the integrals in each flow is independent of $R e_{\lambda}$ and is about 0.5 , illustrating the constancy of $G_{\theta} / R e_{\lambda}$.

The above results suggest that since the term on the right of (14) must eventually vanish at infinitely large Reynolds numbers, then the magnitude of $S_{T}$ should become constant at sufficiently large $R e_{\lambda}$ in decaying grid turbulence. For SFPBT, $-S_{T}$ should be essentially independent of $R e_{\lambda}$, even at low values of $R e_{\lambda}$, since $C_{\theta} \approx 0$. Distributions of $-S_{T}$ vs. $R e_{\lambda}$ in grid turbulence and SFPBT are shown in Fig. 5. Note that $-S_{T}$ is calculated using Eq. (14) with the second term on the left side estimated using the right side of (17), (i.e., by integrating the curves in Fig. 3). As anticipated from our analysis, the magnitude of $-S_{T}$ for SFPBT is virtually independent of $R e_{\lambda}$, with a value of about 0.50 . On the other hand, $-S_{T}$ shows a strong dependence on the Reynolds number in decaying grid turbulence. It starts at a relatively small value, increases as the Reynolds number increases, and eventually approaches a constant $(\simeq 0.50)$ when $R e_{\lambda} \geq 1000$. This behaviour, consistent with the analytical results in Section II, indicates a finite Reynolds number (FRN) effect. ${ }^{9,10}$ Antonia and Burattini ${ }^{9}$ showed that the FRN effect is well reflected in the approach of the third-order velocity structure function to the asymptotic value of $4 / 5$ in the inertial range; the approach is significantly slower in decaying turbulence than when forcing is present.

The FRN effect will very likely differ in different flows. For example, in the far-field of a round jet along jet axis or on the centreline in the two-dimensional cases of a far-wake, the transport equation for $\overline{\theta^{2}}$ can be reasonably well approximated by ${ }^{51-53}$

$$
\frac{1}{2} U \frac{\partial \overline{\theta^{2}}}{\partial x}+\frac{1}{2} \frac{\partial \overline{v \theta^{2}}}{\partial y}+\bar{\epsilon}_{\theta} \approx 0 .
$$

Note that (19) differs from Eq. (9) in grid turbulence through the appearance of the diffusion term. In essence, this difference reflects the different physical processes involved in the transport equation for $\overline{\theta^{2}}$ (large scale) between the grid turbulence and jet axis and wake centreline. The same physical processes should remain at play in the transport equation for $\bar{\epsilon}_{\theta}$ (small scale) in these flows. They translate into different values of the constant $C_{\theta}$ (Eq. (14)). Since $G_{\theta} / R e_{\lambda}$ becomes constant at 


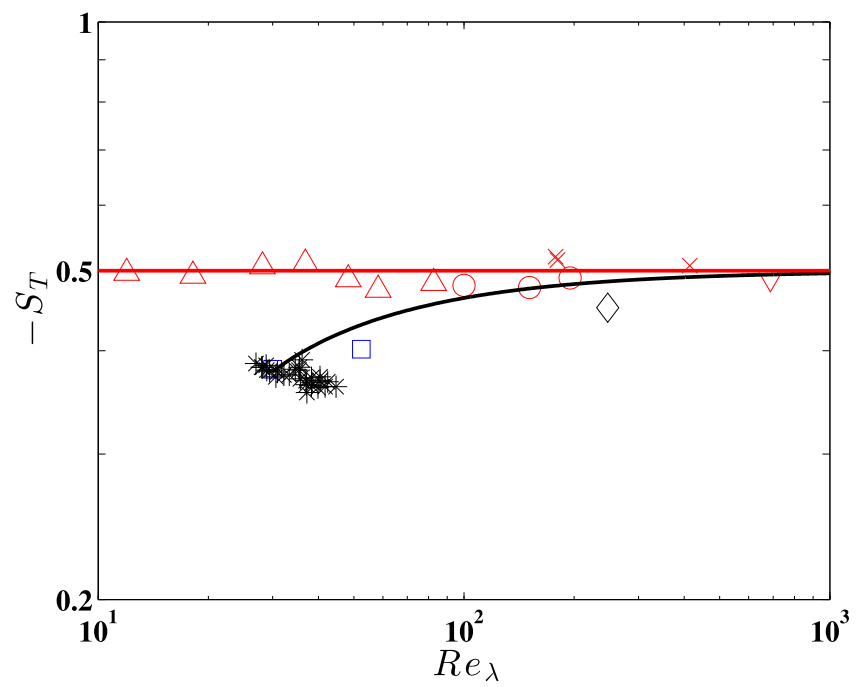

FIG. 5. Dependence of $-S_{T}$ on $R e_{\lambda}$ in grid turbulence: blue square, Antonia et al. $;{ }^{46}$ asterisks, Lee et al. $;{ }^{29}$ diamond, Mydlarski and Warhaft. ${ }^{47}$ The data for SFPBT are also shown for the purpose of comparison: red bigtriangleup, Kerr; ${ }^{23}$ red bigcirc, Wang, Chen, and Brasseur; ${ }^{39}$ red times, Watanabe and $\mathrm{Gotoh}^{38}$ (only the data for $k_{\text {max }}^{*}>1.1$ are shown); red bigtriangledown, Gotoh, Watanabe, and Suzuki. ${ }^{48} 2$ curves (black and red), inferred from Eq. (14), i.e., $S_{T}=\frac{C_{\theta}}{R e_{\lambda}}-\frac{2 \sqrt{15}}{9} \frac{G_{\theta}}{P e} \operatorname{Pr}^{1 / 2} R_{\theta}^{-1 / 2}$, by assuming that $\frac{2 \sqrt{15}}{9} \frac{G_{\theta}}{P e} \operatorname{Pr}^{1 / 2} R_{\theta}^{-1 / 2}=$ constant $\left(\approx 0.50\right.$ for $\left.\operatorname{Re}_{\lambda}>30\right)$, correspond to decaying grid turbulence $(n=m=1.2)$ and SFPBT, respectively.

relatively small $R e_{\lambda}$, one expects that $-S_{T}$ should become constant in the jet axis \& wake centreline, the actual value of $R e_{\lambda}$ at which this occurs depending on the flow and for a given flow, it may also depend on the initial conditions.

In the literature, high $R e_{\lambda}$ atmospheric surface layer (ASL) data have been used for providing support for K62, e.g., Fig. 9 of Ref. 14; the data are replotted in Fig. 6 except that a "red" circle has been drawn around the cluster of symbols which corresponds to the ASL data. Also included

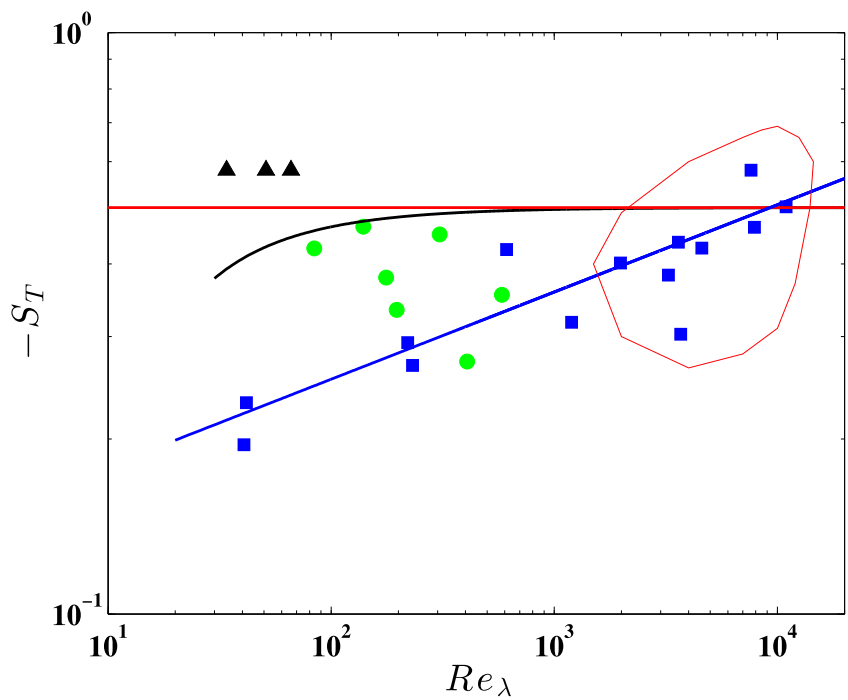

FIG. 6. Dependence of $-S_{T}$ on $R e_{\lambda}$. Blue symbols correspond to Fig. 9 of Sreenivasan and Antonia; ${ }^{14}$ the data sources are not identified here. A "red" circle has been placed around the cluster of data in the ASL to distinguish these data from the remaining (laboratory) data. The blue curve corresponds to $-S_{T} \sim R e_{\lambda}^{0.15}$ (Sreenivasan and Antonia ${ }^{14}$ ). green filled circle, decaying grid turbulence with an imposed mean temperature gradient ${ }^{49}$ black filled triangle, DNS data on the centreline of a fully developed channel flow. ${ }^{50}$ For reference, the 2 curves (black and red) shown in Fig. 5 are reproduced here. 
in Fig. 6 are the 2 curves (black and red), inferred from Eq. (14). Evidently, there is a discrepancy between the ASL data in term of its increasing trend (K62) and $K 41$ (\& O49). We stress that Mydlarski ${ }^{49}$ measured $-S_{T}$ in decaying grid turbulence with an imposed mean temperature gradient over a large range of $\operatorname{Re}_{\lambda}\left(85 \leq R e_{\lambda} \leq 582\right)$ and showed that $-S_{T}$ has no increasing trend $(0.4 \pm 0.1)$. All the data for $\left|-S_{T}\right|$ shown in the work of Sreenivasan and Antonia, ${ }^{14}$ with the exception of Kerr's SFPBT data (Fig. 4), had been previously compiled by Antonia and Chambers. ${ }^{54}$ A major criticism can be levelled at fitting all the data with one straight line and hence a unique dependence $\left(\sim R e_{\lambda}^{0.15}\right)$ on $R e_{\lambda}$ since the data were collected in several different flows, one expects that each flow would exhibit different degrees of departure from local homogeneity and isotropy. More importantly, but in keeping with the previous remark, the effect of the large scales on the small scale motion is likely to differ in each case. Correspondingly, the FRN effect is likely to vary from flow to flow. Ideally, this effect should be estimated, in each flow, using an approach similar to that outlined in Section II for grid turbulence and SFPBT. The upshot of this discussion is that one should really discount the power-law relation $\left(\sim R e_{\lambda}^{0.15}\right)$ since it is meaningless (this comment would apply equally to all previous attempts at fitting data for various normalized velocity and temperature derivative moments in order to establish their dependence on $R e_{\lambda}$ by unique power-law relations). In our previous papers (SA15), dealing with $-S$, it is argued that the ASL data should not have been used in the context of verifying the upper relation of Eq. (3) since these data have most likely been affected by the combined effects of the mean shear and proximity to the surface. Surprisingly, the encircled cluster of ASL data for $-S_{T}$ lies significantly below the corresponding ASL cluster for $-S$ (Fig. 5 of Ref. 55 or Fig. 5 Ref. 14). One would have expected comparable magnitudes of $-S$ and $-S_{T}$ in a neutrally stable ASL. Indeed, the passively heated channel flow DNS distributions of $-S$ and $-S_{T}{ }^{50}$ follow each others closely, almost independently of the location across the channel. The maximum value occurs near the wall; for example, at $h^{+}=640$ (where $h$ is the channel half-width and the superscript denotes normalization by the friction velocity $U_{\tau}$ and $\left.v\right)$, the peak is at $y / h \approx 0.05$ and has a magnitude of about 1.5. In the outer part of the channel $(y / h>0.2),\left|-S_{T}\right|$ (and $|-S|$ ) is approximately constant with a value close to 0.58 , which is somewhat larger than the "asymptotic" value ( 0.50) indicated in Fig. 5 (SA15 showed that the "asymptotic" value for $|-S|$ is 0.53 ). We do not have an explanation for the difference between $-S$ and $-S_{T}$ in the ASL, although for the highest $\operatorname{Re}_{\lambda}\left(\approx 10^{4}\right)$ ASL data point (measured by Antonia and Chambers $\left.{ }^{54}\right), z / L_{M 0}(z$, the distance above the ground, was $4 \mathrm{~m}$; the Monin-Obukhov length scale $-L_{M 0}$ was about $-8 m$ ) was -0.5 , i.e., the conditions far from a neutrally stable state. Naturally, it would be of interest to measure $-S_{T}$ in a neutrally stable ASL, preferably in the range $0.2 \leq z / \delta \leq 0.5$, where $\delta$ is the boundary layer thickness, to confirm that $-S_{T}$ is indeed bounded at values of $R e_{\lambda}$ in excess of $10^{4}$.

\section{CONCLUDING DISCUSSION}

The mixed-derivative skewness, $S_{T}$, in decaying grid turbulence and SFPBT has been investigated through the transport equation for the mean scalar dissipation rate $\bar{\epsilon}_{\theta}$ derived by considering the behaviour of the generalized form of Yaglom's equation at small separation.

An analytical expression (Eq. (14)) relating $-S_{T}$ and the destruction coefficient of $\bar{\epsilon}_{\theta}$ was derived for decaying grid turbulence and SFBPT and tested against experimental (grid turbulence) and direct numerical simulation (SFBPT) data. The available data agree well with the analytical results (Fig. 5). In decaying grid turbulence, $-S_{T}$ approaches a constant value as $R e_{\lambda}$ increases, while for SFPBT, $-S_{T}$ is very nearly constant (i.e., independent of $R e_{\lambda}$ ). Further, it appears that the high Reynolds number value of $-S_{T}$ in grid turbulence is the same as for SFBPT, i.e., $-S_{T} \simeq 0.50$. A similar behaviour for $-S$ vs. $\operatorname{Re}_{\lambda}$ has been reported in various turbulent flows, ${ }^{17,19,20}$ although the asymptotic value of $-S$ appears to be slightly larger than $-S_{T}$. This is consistent with the inequality $\left|-S_{T}\right|<|-S|$ obtained by Lumley ${ }^{56}$ using the Schwarz and similar inequalities. ${ }^{8}$ On the other hand, according to the intermittency models of K62 and O62, where $\alpha$ is in the range $0.09 \leq \alpha \leq 0.12^{57}$ and $\beta=0.15^{14}$ in Eq. (3), $\left|-S_{T}\right|$ would become larger than $|-S|$ as $R e_{\lambda}$ continues to increase; this would violate the inequality $\left|-S_{T}\right|<|-S|{ }^{56}$ 
The results strongly suggest that $-S_{T}$ cannot grow unboundedly as $R e_{\lambda}$ increases and support the argument that $-S_{T}$ approaches an asymptotic value at different rates in different turbulent flows. They are therefore inconsistent with previous work which advocates a unique power-law relation $\left(\sim R e_{\lambda}^{0.15}\right)$ when the ASL data are included. Clearly, this inclusion has biased previous attempts to account for the FRN effect and thus misled researchers into prematurely embracing K62 (O62) as a viable replacement for K41 (O49). Strictly, both K41 and K62 can only be effectively tested in homogeneous isotropic turbulence as $R e_{\lambda} \rightarrow \infty$, viz., the conditions originally stipulated by Kolmogorov. ${ }^{2}$ The present results and those obtained previously ${ }^{17,19,20}$ for the behaviour of $S$, whilst highlighting previous shortcomings in correctly accounting for the FRN effect, are insufficient to allow any definitive conclusion regarding the need to apply intermittency corrections to K41 to be drawn.

\section{ACKNOWLEDGMENTS}

The financial support of the Australian Research Council (ARC) is gratefully acknowledged. Y.Z. wishes to acknowledge support given to him from NSFC through Grant No. 51421063 and from Scientific Research Fund of Shenzhen Government through Grant No. KQCX2014052114430139.

${ }^{1}$ J. C. Wyngaard, “The effect of velocity sensitivity on temperature derivative statistics in isotropic turbulence," J. Fluid Mech. 48, 763-769 (1971).

2 A. N. Kolmogorov, "The local structure of turbulence in incompressible viscous fluid for very large Reynolds number," Dokl. Akad. Nauk SSSR 30, 299-303 (1941b); (see also Proc. R. Soc. Lond. A 434, 9-13 (1991)).

${ }^{3}$ A. M. Oboukhov, "Structure of the temperature field in turbulent flows," Izv. Akad. Nauk. SSSR Geogr. Geofiz 13, 58-69 (1949).

${ }^{4}$ A. N. Kolmogorov, "A refinement of previous hypotheses concerning the local structure of turbulence in a viscous incompressible fluid at high Reynolds number," J. Fluid Mech. 13, 82-85 (1962).

${ }^{5}$ A. M. Obukhov, "Some specific features of atmospheric turbulence," J. Fluid Mech. 13, 77-81 (1962).

${ }^{6}$ A. N. Kolmogorov, "Dissipation of energy in the locally isotropic turbulence," Dokl. Akad. Nauk SSSR 32, 19-21 (1941a); (see also "Turbulence and Stochastic Process: Kolmogorov's ideas 50 years on," Proc.: Math. Phys. Sci. 434, 15-17 (1980)).

${ }^{7}$ A. M. Yaglom, "On the local structure of a temperature field in a turbulent flow," Dokl. Akad. Nauk SSSR 69, 743-746 (1949).

${ }^{8}$ A. S. Monin and A. M. Yaglom, Statistical Fluid Dynamics (MIT, 2007), Vol. 2.

${ }^{9}$ R. A. Antonia and P. Burattini, "Approach to the 4/5 law in homogeneous isotropic turbulence," J. Fluid Mech. 550, 175-184 (2006).

10 V. L'vov and I. Procaccia, "Intermittency in hydrodynamic turbulence as intermediate asymptotics to Kolmogorov scaling," Phys. Rev. Lett. 74, 2690 (1995).

${ }^{11}$ J. Qian, "Inertial range and the finite Reynolds number effect of turbulence," Phys. Rev. E 55, 337-342 (1997).

12 J. Qian, "Normal and anomalous scaling of turbulence," Phys. Rev. E 58, 7325 (1998).

${ }^{13}$ S. L. Tang, R. A. Antonia, L. Djenidi, L. Djenidi, and Y. Zhou, "Finite Reynolds number effect on the scaling range behavior of turbulent longitudinal velocity structure functions," J. Fluid Mech. (submitted).

${ }^{14}$ K. Sreenivasan and R. A. Antonia, "The phenomenology of small-scale turbulence," Annu. Rev. Fluid Mech. 29, 435-472 (1997).

15 Z. Warhaft, "Passive scalars in turbulent flows," Annu. Rev. Fluid Mech. 32, 203-240 (2000).

${ }^{16}$ P. Chassaing, R. A. Antonia, F. Anselmet, L. Joly, and S. Sarkar, Variable Density Fluid Turbulence (Springer Science \& Business Media, 2013).

${ }^{17}$ R. A. Antonia, S. L. Tang, L. Djenidi, and L. Danaila, "Boundedness of the velocity derivative skewness in various turbulent flows," J. Fluid Mech. 781, 727-744 (2015).

${ }^{18}$ F. Thiesset, R. A. Antonia, and L. Djenidi, “Consequences of self-preservation on the axis of a turbulent round jet,” J. Fluid Mech. 748, 11 (2014).

${ }^{19}$ S. L. Tang, R. A. Antonia, L. Djenidi, H. Abe, T. Zhou, L. Danaila, and Y. Zhou, “Transport equation for the mean turbulent energy dissipation rate on the centreline of a fully developed channel flow," J. Fluid Mech. 777, 151-177 (2015).

${ }^{20}$ S. L. Tang, R. A. Antonia, L. Djenidi, and Y. Zhou, "Transport equation for the isotropic turbulent energy dissipation rate in the far-wake of a circular cylinder," J. Fluid Mech. 784, 109-129 (2015).

${ }^{21}$ J. Qian, "Skewness factor of turbulent velocity derivative," Acta Mech. Sin. 10, 12-15 (1994).

${ }^{22}$ R. A. Antonia, L. Djenidi, and L. Danaila, "Collapse of the turbulent dissipation range on Kolmogorov scales," Phys. Fluids 26, 045105 (2014).

${ }^{23}$ R. M. Kerr, "Higher-order derivative correlations and the alignment of small-scale structures in isotropic numerical turbulence," J. Fluid Mech. 153, 31-58 (1985).

${ }^{24}$ L. Danaila, F. Anselmet, T. Zhou, and R. A. Antonia, "A generalization of Yaglom's equation which accounts for the large-scale forcing in heated decaying turbulence," J. Fluid Mech. 391, 359-372 (1999).

${ }^{25}$ L. Danaila and L. Mydlarski, "Effect of gradient production on scalar fluctuations in decaying grid turbulence," Phys. Rev. E 64, 016316 (2001). 
${ }^{26}$ L. Djenidi and R. A. Antonia, "A general self-preservation analysis for decaying homogeneous isotropic turbulence," J. Fluid Mech. 773, 345-365 (2015).

${ }^{27}$ L. Djenidi, M. Kamruzzaman, and R. A. Antonia, "Power-law exponent in the transition period of decay in grid turbulence," J. Fluid Mech. 779, 544-555 (2015).

${ }^{28}$ C. G. Speziale and P. S. Bernard, "The energy decay in self-preserving isotropic turbulence revisited," J. Fluid Mech. 241, 645-667 (1992).

${ }^{29}$ S. K. Lee, L. Djenidi, R. A. Antonia, and L. Danaila, "On the destruction coefficients for slightly heated decaying grid turbulence,” Int. J. Heat Fluid Flow 43, 129-136 (2013).

${ }^{30}$ D. Fukayama, T. Oyamada, T. Nakano, T. Gotoh, and K. Yamamoto, "Longitudinal structure functions in decaying and forced turbulence," J. Phys. Soc. Jpn. 69, 701-715 (2000).

${ }^{31}$ T. Gotoh, D. Fukayama, and T. Nakano, "Velocity field statistics in homogeneous steady turbulence obtained using a high-resolution direct numerical simulation," Phys. Fluids 14, 1065-1081 (2002).

${ }^{32} \mathrm{~S}$. Corrsin, "On the spectrum of isotropic temperature fluctuations in an isotropic turbulence," J. Appl. Phys. 22, 469-473 (1951).

${ }^{33} \mathrm{P}$. K. Yeung, S. Xu, and K. R. Sreenivasan, "Schmidt number effects on turbulent transport with uniform mean scalar gradient," Phys. Fluids 14, 4178-4191 (2002).

${ }^{34}$ R. A. Antonia and P. Orlandi, "Effect of Schmidt number on small-scale passive scalar turbulence," Appl. Mech. Rev. 56, 615-632 (2003).

${ }^{35}$ D. A. Donzis, K. R. Sreenivasan, and P. K. Yeung, "The Batchelor spectrum for mixing of passive scalars in isotropic turbulence," Flow, Turbul. Combust. 85, 549-566 (2010).

${ }^{36}$ T. Gotoh and T. Watanabe, "Scalar flux in a uniform mean scalar gradient in homogeneous isotropic steady turbulence," Physica D 241, 141-148 (2012).

${ }^{37}$ T. Watanabe and T. Gotoh, "Statistics of a passive scalar in homogeneous turbulence," New J. Phys. 6, 40 (2004).

${ }^{38} \mathrm{~T}$. Watanabe and T. Gotoh, "Inertial-range intermittency and accuracy of direct numerical simulation for turbulence and passive scalar turbulence," J. Fluid Mech. 590, 117-146 (2007).

${ }^{39}$ L.-P. Wang, S. Chen, and J. G. Brasseur, "Examination of hypotheses in the Kolmogorov refined turbulence theory through high-resolution simulations. II. Passive scalar field," J. Fluid Mech. 400, 163-197 (1999).

${ }^{40}$ M. Gauding, "Statistics and scaling laws of turbulent scalar mixing at high Reynolds numbers," Ph.D. thesis, RWTH Aachen University, 2014.

${ }^{41}$ R. A. Antonia, R. J. Smalley, T. Zhou, F. Anselmet, and L. Danaila, "Similarity solution of temperature structure functions in decaying homogeneous isotropic turbulence," Phys. Rev. E 69, 016305 (2004).

${ }^{42}$ S. K. Lee, A. Benaissa, L. Djenidi, P. Lavoie, and R. A. Antonia, "Scaling range of velocity and passive scalar spectra in grid turbulence," Phys. Fluids 24, 075101 (2012).

43 A. Berajeklian and L. Mydlarski, "Simultaneous velocity-temperature measurements in the heated wake of a cylinder with implications for the modeling of turbulent passive scalars," Phys. Fluids 23, 055107 (2011).

${ }^{44}$ N. Lefeuvre, L. Djenidi, R. A. Antonia, and T. Zhou, "Turbulent kinetic energy and temperature variance budgets in the far-wake generated by a circular cylinder," in 19th Australasian Fluid Mechanics Conference, Melbourne, Australia, 8-11 December 2014.

${ }^{45} \mathrm{H}$. Abe, R. A. Antonia, and H. Kawamura, "Correlation between small-scale velocity and scalar fluctuations in a turbulent channel flow," J. Fluid Mech. 627, 1-32 (2009).

${ }^{46}$ R. A. Antonia, T. Zhou, L. Danaila, and F. Anselmet, “Streamwise inhomogeneity of decaying grid turbulence,” Phys. Fluids 12, 3086 (2000).

${ }^{47}$ L. Mydlarski and Z. Warhaft, "Passive scalar statistics in high-Peclet-number grid turbulence," J. Fluid Mech. 358, 135-175 (1998).

${ }^{48}$ T. Gotoh, T. Watanabe, and Y. Suzuki, "Universality and anisotropy in passive scalar fluctuations in turbulence with uniform mean gradient," J. Turbul. 12, 1-27 (2011).

${ }^{49}$ L. Mydlarski, "Mixed velocity-passive scalar statistics in high-Reynolds-number turbulence," J. Fluid Mech. 475, 173-203 (2003).

${ }^{50}$ R. A. Antonia and H. Abe, "Analogy between small-scale velocity and passive scalar fields in a turbulent channel flow," in Euromech Colloquium (Acad. Sci. Torino, Torino, Italy, 2009), Vol. 512, pp. 26-29.

${ }^{51}$ R. A. Antonia and L. W. Browne, "Anisotropy of temperature dissipation in a turbulent wake," J. Fluid Mech. 163, 393-403 (1986).

52 R. Antonia and J. Mi, “Temperature dissipation in a turbulent round jet," J. Fluid Mech. 250, 531-551 (1993).

${ }^{53}$ A. Darisse, J. Lemay, and A. Benaïssa, "Extensive study of temperature dissipation measurements on the centerline of a turbulent round jet based on the $\overline{\theta^{2}} / 2$ budget," Exp. Fluids 55, 1-15 (2014).

${ }^{54}$ R. A. Antonia and A. J. Chambers, "On the correlation between turbulent velocity and temperature derivatives in the atmospheric surface layer," Boundary-Layer Meteorol. 18, 399-410 (1980).

55 J. C. Wyngaard and H. Tennekes, "Measurements of the small-scale structure of turbulence at moderate Reynolds numbers," Phys. Fluids 13, 1962-1969 (1970).

56 J. L. Lumley, "On third-order mixed moments," Phys. Fluids 17, 1127-1129 (1974)

${ }^{57}$ T. Ishihara, Y. Kaneda, M. Yokokawa, K. Itakura, and A. Uno, "Small-scale statistics in high-resolution direct numerical simulation of turbulence: Reynolds number dependence of one-point velocity gradient statistics," J. Fluid Mech. 592, 335-366 (2007). 